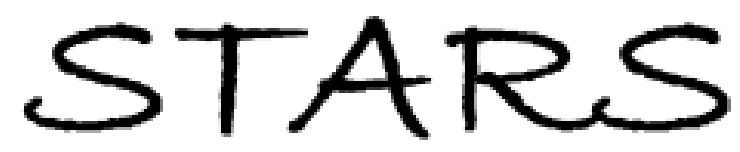

University of Central Florida

STARS

$1-1-2014$

\title{
Theoretical study of two-photon circular dichroism on molecular structures simulating aromatic amino acid residues in proteins with secondary structures
}

Yuly Vesga

University of Central Florida

Carlos Diaz

University of Central Florida

Florencio E. Hernandez

University of Central Florida

Find similar works at: https://stars.library.ucf.edu/facultybib2010

University of Central Florida Libraries http://library.ucf.edu

This Article is brought to you for free and open access by the Faculty Bibliography at STARS. It has been accepted for inclusion in Faculty Bibliography 2010s by an authorized administrator of STARS. For more information, please contact STARS@ucf.edu.

\section{Recommended Citation}

Vesga, Yuly; Diaz, Carlos; and Hernandez, Florencio E., "Theoretical study of two-photon circular dichroism on molecular structures simulating aromatic amino acid residues in proteins with secondary structures" (2014). Faculty Bibliography 2010s. 6231.

https://stars.library.ucf.edu/facultybib2010/6231

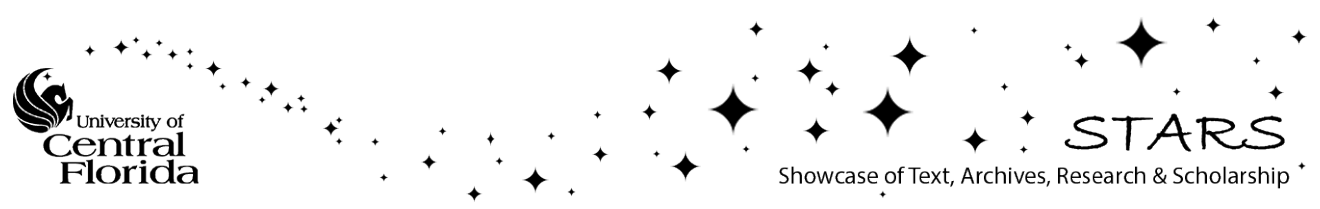




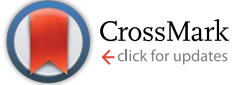

Cite this: RSC Adv., 2014, 4, 60974

Received 8th August 2014

Accepted 10th November 2014

DOI: $10.1039 / \mathrm{c} 4 \mathrm{ra0} 8383 \mathrm{k}$

www.rsc.org/advances

\section{Theoretical study of two-photon circular dichroism on molecular structures simulating aromatic amino acid residues in proteins with secondary structures $\uparrow$}

\author{
Yuly Vesga, ${ }^{a}$ Carlos Diaz ${ }^{a}$ and Florencio E. Hernandez ${ }^{\star a b}$ \\ Herein, we report on the calculation and the comparative analysis of the theoretical two-photon circular \\ dichroism (TPCD) spectra of L-histidine (His), L-phenylalanine (Phe), and L-tyrosine (Tyr) simulating \\ residues in proteins with secondary structures ( $\alpha$-helix, $\beta$-strand and random coil), down to the far-UV \\ region (FUV). This work exposes unique signatures in the FUV for each conformer in each configuration. \\ The outcomes of this research show how FUV-TPCD can be used to study peptide and protein \\ structures in a region never evaluated before but packed with important structural information.
}

\section{Introduction}

Electronic circular dichroism (ECD) has played a major role in the study of the conformational and physical-chemical properties of optically active biomolecules such as peptides and proteins over the last several years., ${ }^{\mathbf{1 2}}$ Determining relevant features of the structure of these biomolecules and the exchange between them and with their environment is possible by the presence of chromophores that can convey a clear ECD signal. Distinctive absorption bands ( $n-\pi^{*}$ and $\pi-\pi^{*}$ transitions) in the near-ultraviolet (NUV) and far-ultraviolet (FUV) regions are provided by peptide bonds of secondary protein structures in $\alpha$-helix, $\beta$-strand and random coil configurations. ${ }^{3-5}$ Aromatic amino acid side-chains of peptides in the same spectral region produce a signal that offers important information about the rigidity and environment of these structures. ${ }^{6-8}$ Even though ECD is a trustworthy technique for the study of proteins in the UV, this method presents some inevitable underlying weaknesses, i.e. the contribution of aromatic amino acid side-chain chromophores to ECD bands in the NUV inhibits the analysis of the secondary structure of the main-chain of peptides and proteins. ${ }^{9}$

In order to help overcome this hindrance and estimate the contribution of aromatic side-chains to the UV region of the ECD spectra of proteins, in 2006 Kodama and co-workers ${ }^{\mathbf{1 0}}$ reproduced the ECD spectra of various side-chain conformations of model compounds. They used density functional theory $(\mathrm{DFT})^{\mathbf{1 1}}$ and Ramachandran diagrams ${ }^{\mathbf{1 2}}$ to create the backbone

${ }^{a}$ Department of Chemistry, University of Central Florida, P. O. Box 162366, Orlando, Florida 32816-2366, USA. E-mail: florencio.hernandez@ucf.edu

${ }^{b}$ The College of Optics and Photonics, CREOL, University of Central Florida, P. O. Box 162366, Orlando, Florida 32816-2366, USA

$\dagger$ Electronic supplementary information (ESI) available. See DOI: $10.1039 / \mathrm{c} 4 \mathrm{ra} 08383 \mathrm{k}$ dihedral angles $\psi$ and $\phi$ of amino acid residues in proteins, optimizing the structures of model compounds in these biological molecules. Their calculations established that the ECD spectra of the aromatic residue models in $\alpha$-helix, $\beta$-strand and random-coil configurations depend on the main-chain and the side-chain conformations. ${ }^{12}$ Nevertheless, because some of the aromatic amino acids exhibit a complex ECD pattern in the NUV region, they concluded that it is very challenging to distinguish different conformers in this region. With the purpose of surmounting this barrier, scientists have been attempting to access the FUV using Synchrotron radiation CD. ${ }^{9}$ However, this method presents clear shortcomings in this spectral region because it is still based on the one-photon absorption (OPA) of optically active compounds. The OPA of amino acids typically occurs in the NUV (380-200 $\mathrm{nm}$ ) to FUV (200-100 nm) region of the electromagnetic spectrum. As a result, the OPA of standard aqueous buffers in the same spectral region covers the small ECD signal and the scattering present at shorter wavelengths becomes an obstacle in heterogeneous samples. This has made further investigations of these essential biological systems more challenging.

To circumvent the characteristic limitations of ECD, Hernandez and co-workers recently proposed to utilize two-photon circular dichroism (TPCD), ${ }^{13}$ which is a nonlinear spectroscopic technique analogous to ECD. First proposed theoretically ${ }^{\mathbf{1 4 - 1 6}}$ in the 1970s and invigorated in 2005, ${ }^{17}$ TPCD facilitates the examination of chiral structures in the spectral region of the FUV that is inaccessible via ECD. TPCD is defined as the difference between the two-photon absorption (TPA) crosssections for two photons of the same energy but opposite circular polarization (left and right). ${ }^{\mathbf{1 4 - 1 6}}$ The measurement of this property was recently made possible with the establishment of the reliable double L-scan technique developed by Hernandez et al. ${ }^{18}$ 
Since TPA processes are generated at longer wavelengths than those of OPA, ${ }^{19}$ the OPA in the TPA excitation region is usually insignificant and the scattering is greatly reduced. Furthermore, the existent quadratic dependence with the incident irradiance of TPA grants this excitation process more spatial resolution and penetration depth. This dependence also provides enhanced background discrimination and reduced photodamage to living specimens. ${ }^{20}$ Vast technological applications in the fields of bioimaging ${ }^{20,21}$ and photodynamic therapy $^{22}$ have been uncovered by these particular attributes. Moreover, TPCD is especially sensitive to small peptide structural distortions like variances in Ramachandran dihedral angles ${ }^{12}$ and bond lengths of standard amino acids as demonstrated by Agren et al., ${ }^{23}$ and even more recently by Hernandez and co-workers. ${ }^{24}$ Thus, TPCD seems to be a harmonizing and favorable approach for the in-depth study and analysis of biomolecules in vitro.

As a continuation of our first work on TPCD of molecular structures simulating L-tryptophan residues in proteins, ${ }^{24}$ our goal here is to determine the contribution of side- and mainchains conformation of aromatic amino acid residues to the TPCD spectra of proteins, in the NUV and FUV region. In order to accomplish this objective, we have embarked on the systematic and advanced conformational analysis of the theoretical TPA and TPCD spectra for a variety of side-chain conformations on model compounds in their secondary structure configuration, i.e. $\alpha$-helix, $\beta$-strand and random coil.

In this article we report on the calculation and analysis of the theoretical TPA and TPCD of molecular structures imitating phenylalanine (Phe), tyrosine (Tyr), and histidine (His) residues in proteins with secondary structure configurations. The comparative examination of the TPCD spectra of the various conformers in each configuration exposes unique fingerprints down to the FUV, a blind spectral region for ECD. Results exhibited in this article confirm the potential of FUV-TPCD to identify and study the structures of proteins in a region where intrinsic solvent absorption and sample scattering cover the ECD signal.

\section{Theoretical and computational methods}

The molecular structure of the L-stereoisomers of the main six histidine residues (His11, His12, His21, His22, His31 and His32), the six primary tyrosine residues (Tyr11, Tyr12, Tyr21, Tyr22, Tyr31 and Tyr32), and the three principal phenylalanine residues (Phe1, Phe2 and Phe3), were optimized using Density Functional Theory (DFT), ${ }^{\mathbf{1 1}, 25}$ employing the Becke's threeparameter exchange, Lee, Yang and Parr correlation (B3LYP) hybrid functional ${ }^{26-28}$ in combination with the 6-311G(d) basis set, ${ }^{29,30}$ employing Gaussian 09. ${ }^{31}$ The establishment and optimization of all the His, Tyr and Phe conformers, in their corresponding $\alpha$-helix, $\beta$-strand and random coil configuration, were achieved using initially the well-known Ramachandran dihedral angles for such structures. ${ }^{12}$ These angles account for the relative configuration of the two groups about the $\mathrm{C}_{\alpha}$ atom which are specified by $\psi$ and $\phi$ (main-chain angles). The relative positioning of these two groups with respect to the $\mathrm{C}_{\alpha}-\mathrm{C}_{\beta}$ and $\mathrm{C}_{\beta}-\mathrm{C}_{\gamma}$ bonds were estimated based on the side-chain angles $\chi_{1}$ and $\chi_{2}$ as shown in ref. 24. Main- and side-chain angle values for all three amino acids were extracted from ref. 10 (see Fig. 1 in ref. 24).

TPA and TPCD for the lowest 80 electronic excited states of all optimized structures were calculated using Time-Dependent DFT (TD-DFT), ${ }^{11,25}$ using B3LYP and the Coulomb Attenuated Method variant of B3LYP (CAM-B3LYP) exchange correlation functionals (XCF), and the 6-311G(d) basis set ${ }^{29,30}$ using Dalton $2011 .^{32}$ All the calculations were completed in gas phase.

The TPA spectra were calculated using eqn (1). ${ }^{33-36}$

$$
\delta_{0 f}^{\mathrm{TPA}}(\omega) \approx 1.25273 \times 10^{-2} \times \omega^{2} \sum_{f} g\left(2 \omega, \omega_{0 f}, \Gamma\right) \cdot \bar{\delta}_{0 f}^{\mathrm{TPA}}\left(\omega_{0 f}\right) .
$$

Here $\delta_{0 f}^{\mathrm{TPA}}\left(\omega_{0 f}\right)$ is the orientational averaged two-photon probability for the degenerate case, $\omega$ is the excitation frequency and $g\left(2 \omega, \omega_{0 f}, \Gamma\right)$ is a normalized Lorentzian lineshape function where $\Gamma$ is the linewidth. The TPA spectra obtained from eqn (1) are in Göppert-Mayer units (GM), i.e. $10^{-50} \mathrm{~cm}^{4} \mathrm{~s}$ per molec per photon when atomic units are used for the elements of eqn (1).

TPCD spectra were computed employing: ${ }^{14,15,36}$

$$
\Delta \delta^{\mathrm{TPCD}}(\omega)=\frac{4}{15} \frac{(2 \pi)^{3}}{c_{0}{ }^{3}\left(4 \pi \varepsilon_{0}\right)^{2}} \times \omega^{2} \sum_{f} g\left(2 \omega, \omega_{0 f}, \Gamma\right) \cdot R_{0 f}^{\mathrm{TPCD}}\left(\omega_{0 f}\right)
$$

$\Delta \delta^{\mathrm{TPCD}}(\omega) \approx 4.87555 \times 10^{-5} \times \omega^{2} \sum_{f} g\left(2 \omega, \omega_{0 f}, \Gamma\right) \cdot R_{0 f}^{\mathrm{TPCD}}\left(\omega_{0 f}\right)$,

where $g\left(2 \omega, \omega_{0}, \Gamma\right)$ is a normalized Lorentzian lineshape function $(\Gamma)$ and $\Delta \delta^{\mathrm{TPCD}}(\omega)$ from eqn (2) is obtained in GM units when the TPCD rotational strength $R_{0 f}^{\mathrm{TPCD}}\left(\omega_{0 f}\right)$ and the circular frequency $\omega$ are in atomic units. $R_{0 f}^{\mathrm{TPCD}}\left(\omega_{0 f}\right)$ is obtained, in atomic units, from:

$$
R_{0 f}^{\mathrm{TPCD}}\left(\omega_{0 f}\right)=-b_{1} B_{1}^{\mathrm{TI}}\left(\omega_{0 f}\right)-b_{2} B_{2}^{\mathrm{TI}}\left(\omega_{0 f}\right)-b_{3} B_{3}^{\mathrm{TI}}\left(\omega_{0 f}\right)
$$

$b_{1}, b_{2}$ and $b_{3}$ are scalars defined by the experimental setup. In order to consider typical experimental conditions, ${ }^{13}$ we have used two left or two right circularly polarized photons propagating collinearly in the same direction, therefore $b_{1}=6, b_{2}=2$ and $b_{3}=-2 .{ }^{\mathbf{1 4 1 5 , 3 6}}$ The molecular parameters $B_{1}, B_{2}$ and $B_{3}$ are acquired from the subsequent equations:

$$
\begin{aligned}
& B_{1}^{\mathrm{TI}}\left(\omega_{0 f}\right)=\frac{1}{\omega^{3}} \sum_{\rho \sigma} M_{\rho \sigma}^{p, 0 f}\left(\omega_{0 f}\right) P_{\rho \sigma}^{p^{*}, 0 f}\left(\omega_{0 f}\right), \\
& B_{2}^{\mathrm{TI}}\left(\omega_{0 f}\right)=\frac{1}{2 \omega^{3}} \sum_{\rho \sigma} T_{\rho \sigma}^{+, 0 f}\left(\omega_{0 f}\right) P_{\rho \sigma}^{p^{*}, 0 f}\left(\omega_{0 f}\right), \\
& B_{3}^{\mathrm{TI}}\left(\omega_{0 f}\right)=\frac{1}{\omega^{3}} \sum_{\rho \sigma} M_{\rho \sigma}^{p, 0 f}\left(\omega_{0 f}\right) P_{\sigma \sigma}^{p^{*}, 0 f}\left(\omega_{0 f}\right) .
\end{aligned}
$$


Here, the generalized two-photon tensors $P_{\rho \sigma}^{p^{*}, 0 f}\left(\omega_{0 f}\right)$ and $T_{\rho \sigma}^{+, 0 f}\left(\omega_{0 f}\right)$ depend on the electric transition dipole and quadrupole matrix elements in the velocity formulation, respectively, and $M_{\rho \sigma}^{p, 0 f}\left(\omega_{0 f}\right)$ is controlled by the magnetic transition dipole matrix elements. ${ }^{\mathbf{1 4 , 1 5 , 3 6}}$

In order to achieve some initial validation of our theoretical approximation we performed linear calculations of the ECD spectra of three of the few amino acids that have been measured down to $150 \mathrm{~nm},{ }^{37}$ i.e. L-Val, L-Leu and L-Ala (see ESI $\dagger$ ). Even though we are mindful that this is not totally suitable to make a definite choice of a preferred XCF, in some cases it can be helpful. ${ }^{38}$

Briefly, OPA and ECD for the lowest 80 electronic excited states were calculated on all optimized structures at the B3LYP/ 6-311G(d) and CAM-B3LYP/6-311G(d) levels of theory using Gaussian 09 (ref. 31) in gas phase (more details are given in $\mathrm{ESI} \dagger$ ). All convoluted spectra were calculated using $\Gamma=0.124 \mathrm{eV}$ (FWHM) in agreement with ref. 10. As determined in previous works, ${ }^{38}$ CAM-B3LYP predicts high energy, long range and charge transfer diffuse states better than B3LYP because the HOMO energy, determined with this XCF, is normally lower than for B3LYP. ${ }^{38}$ Therefore, based on previous studies ${ }^{24}$ we are convinced that CAM-B3LYP should predict more precisely the TPCD spectra of His, Tyr, and Phe residues in the FUV. Nevertheless, we are aware that using this specific XCF is not enough to perform the most accurate calculation because the theoretical results are impacted by the strong state diffusion within the short wavelength region of the spectra, the high density of states and the prospect of intermediate state resonances. For that reason, one should consider complex damped response theory when working with molecules such as His, Tyr, and Phe, ${ }^{39,40}$ which gives the same results as standard response theory if the response approach is able to converge all requested states but the damped response theory approach may prove to be a more computationally convenient way to obtain high-energy states. Unfortunately, this approach is available for $\mathrm{TPA}^{\mathbf{4 1}}$ but not for TPCD yet. On the other hand, because one cannot completely ignore the theoretical results achieved with B3LYP until the experimental TPCD spectra of these amino acids are measured in the FUV, in the ESI $\dagger$ we present all the theoretical TPA and TPCD spectra of His, Tyr, and Phe residues obtained with this XCF.

\section{Results and discussion}

In order to show the observed differences between configurations, the optimized stereochemical structures of His, Tyr, and Phe residues in random coil are presented in Fig. 1. Similar types of structures for the $\alpha$-helix and $\beta$-strand are shown in ESI. $\dagger$ All the optimized angles for each conformer are presented in Tables 1-3. In these three tables, one can observe rather important differences in the main-chain angles for different configurations of His, Tyr, and Phe residues within each conformation. This indicates that, as it was concluded for Trp, ${ }^{24}$ the main-chain angles are unquestionably modified by the sidechain configuration and vice versa. It is also noticeable that the differences in $\psi$ and $\phi$ are more significant in the $\beta$-strand conformation. All these distortions can be explained in terms of the allowable van der Waals distances which demarcate the areas of permissible $\psi$ and $\phi .^{12}$ In di-, tri-, and polypeptide chains, $\psi$ and $\phi$ configurations always take place inside the outer boundaries of the minimum contact distance assumed, and a clustering configuration can emerge. Every time the $\mathrm{C}_{\alpha}$ atom under analysis belongs to a glycyl residue the configuration is close to $180^{\circ}$ and 0 , and whenever there is a $C_{\beta}$ atom the clustering is nearly $150^{\circ}$ and $120^{\circ} . .^{12}$

After having optimized the structures of all residues of the left-handed isomers of His, Tyr, and Phe, in each backbone configuration ( $\alpha$-helix, $\beta$-strand and random coil), we computed their TPA and TPCD spectra within the two-photon excitation wavelength range comprising $220 \mathrm{~nm}$ to $500 \mathrm{~nm}$ (in the OPA scale this range corresponds to a wavelength range from $110 \mathrm{~nm}$ to $250 \mathrm{~nm})$. The convoluted spectra were calculated using CAM-B3LYP/6-311G(d) in Dalton 2011 and with $\Gamma=0.124 \mathrm{eV}$ (FWHM) (theoretical spectra calculated with B3LYP/6-311G(d) can be found in $\mathrm{ESI} \dagger$ ).

First, we examine the effect of the side-chains onto the TPA and TPCD spectra of His, Phe, and Tyr residues, in their corresponding $\beta$-strand, $\alpha$-helix, and random coil configurations (see Fig. 2-8).

In the case of His (Fig. 2-4), the straight comparison between the corresponding spectra of a pair of conformers with equal $\chi_{1}$ uncovers moderately mild differences for TPA, in contrast to considerably big variations in TPCD. The largest difference in TPA is noticed between His11 and His12 in $\beta$-strand, which exhibits a noteworthy variation in the peak located at $252 \mathrm{~nm}$. The fact that TPCD is a technique recognized for its sensitivity to small peptide structural distortions such as side-chain conformational angles and bond lengths of residues, ${ }^{23}$ and Ramachandran dihedral angles, ${ }^{12}$ can be certified one more time by the occurrence of bands with opposite signs (spectral signatures) among residues of His. For instance, between His11 and His12 at $328 \mathrm{~nm}$ and $380 \mathrm{~nm}$, and between His31 and His32 within the spectral region $280-350 \mathrm{~nm}$, there are clear variations in $\beta$-strand. In $\alpha$-helix and random coil, the residues of His look very similar. This makes it challenging to distinguish between a pair of conformers with same $\chi_{1}$. These TPCD spectra also present some exceptions, specifically in His31 and His32 in $\alpha$-helix within a wavelength range of $300-340 \mathrm{~nm}$ as well as in His11 and His12 in the region between 240 and $260 \mathrm{~nm}$ in random coil, where the TPCD signal acquires an opposite sign.

With regard to Phe, in Fig. 5 we can see differences in the TPA and TPCD spectra of the three conformers in each configuration. First, in the TPA spectra of all the conformers in $\beta$-strand, $\alpha$-helix, and Random coil one can distinguish the following different spectral features between 280 and $340 \mathrm{~nm}$ : (i) $\beta$-strand: a strong band centered at $330 \mathrm{~nm}$ for Phe1 and two strong bands at approximately $300 \mathrm{~nm}$ and $340 \mathrm{~nm}$ for Phe2, (ii) $\alpha$-helix: a strong band centered at $\sim 300 \mathrm{~nm}$ for Phe2, and (iii) Random coil: a double band with peaks at $c a .300 \mathrm{~nm}$ and $315 \mathrm{~nm}$ for Phe1. Second, the TPCD spectra of this amino acid in $\beta$-strand reveals evident differences in spectral signatures between the three residues, i.e. alternating sign signals for each conformer within the wavelength range between $300 \mathrm{~nm}$ to 

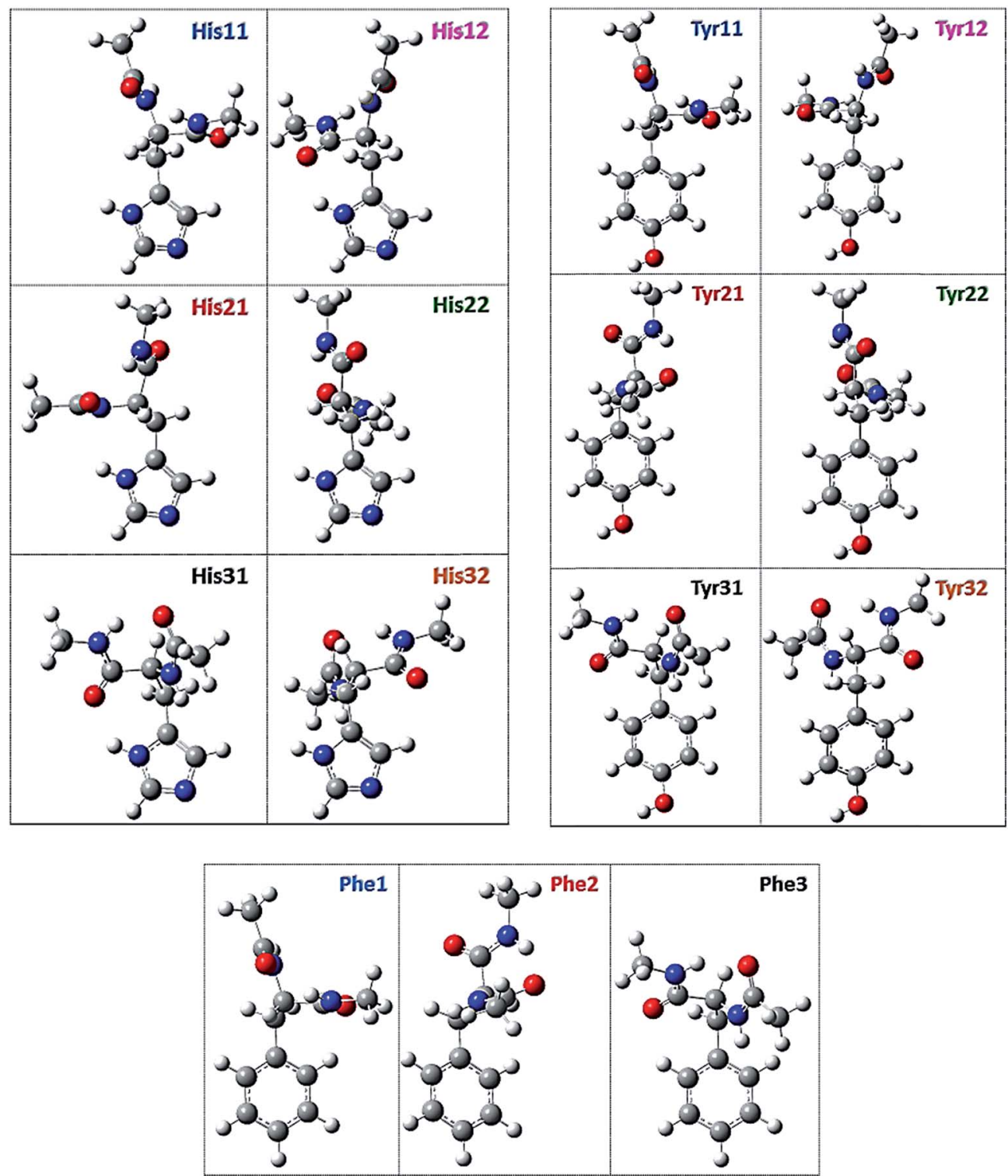

Fig. 1 Stereochemical structures of L-histidine (left), L-tyrosine (right), and L-phenylalanine (down) models in a random coil configuration. Optimizations were performed with DFT/B3LYP/6-311G(d) in gas phase using Gaussian 09. ${ }^{31}$

$380 \mathrm{~nm}$. In $\alpha$-helix, though, the situation is slightly different, while Phe1 is distinguishable from the rest of the residues through specific negative sign features observed at $250 \mathrm{~nm}$ and $280 \mathrm{~nm}$, in Phe 2 the differentiation is more obvious at $c a$. $300 \mathrm{~nm}$ where the sign of the characteristic TPCD spectra is opposite for analogous conformers. The same trend can be notorious in random coil where the identification of the Phe1 residue is possible through its opposite sign TPCD signals between $260 \mathrm{~nm}$ and $290 \mathrm{~nm}$.

Concerning Tyr (Fig. 6-8), it is interesting to highlight the fact that the TPA spectra of all its residues are virtually identical. Likewise, the TPCD spectra of Tyr residues in $\beta$-strand and Random coil are very similar between pairs of conformers. However, the TPCD spectra of equivalent conformers in $\alpha$-helix reveals small but noticeable differences between Tyr11 and
Tyr12, and Tyr31 and Tyr32 - distinct peaks between $300 \mathrm{~nm}$ and $380 \mathrm{~nm}$.

In summary, one can close this part of the analysis by recognizing that TPCD, contrary to standard $\mathrm{ECD},{ }^{\mathbf{1 0}}$ allows us to distinguish between pairs of conformers of amino acid residues in different secondary protein configurations, mainly in His. Through the analysis of the OPA and ECD spectra of residues of similar amino acids performed by Kodama and co-authors, ${ }^{\mathbf{1 0}}$ it has been determined that the aromatic ring present in Phe and Tyr is not anisotropically polarizable as it is in His. Therefore, distinguishing between residues of Phe and Tyr using linear spectroscopy is extremely challenging.

Afterward, we analyzed the effect of the main-chains onto the TPA and TPCD spectra of His, Phe, and Tyr residues in $\alpha$-helix, $\beta$-strand, and random coil conformations. In Fig. 9-11, we 
Table 1 Optimized Ramachandran dihedral angles $(\psi$ and $\phi)$ for the main-chain amide group in L-histidine and the corresponding angles $\left(\chi_{1}\right.$ and $\chi_{2}$ ) for the rotational conformers defining the residues (all in degree). Angles are reported for His residues in random coil, $\alpha$-helix, $\beta$-strand configuration

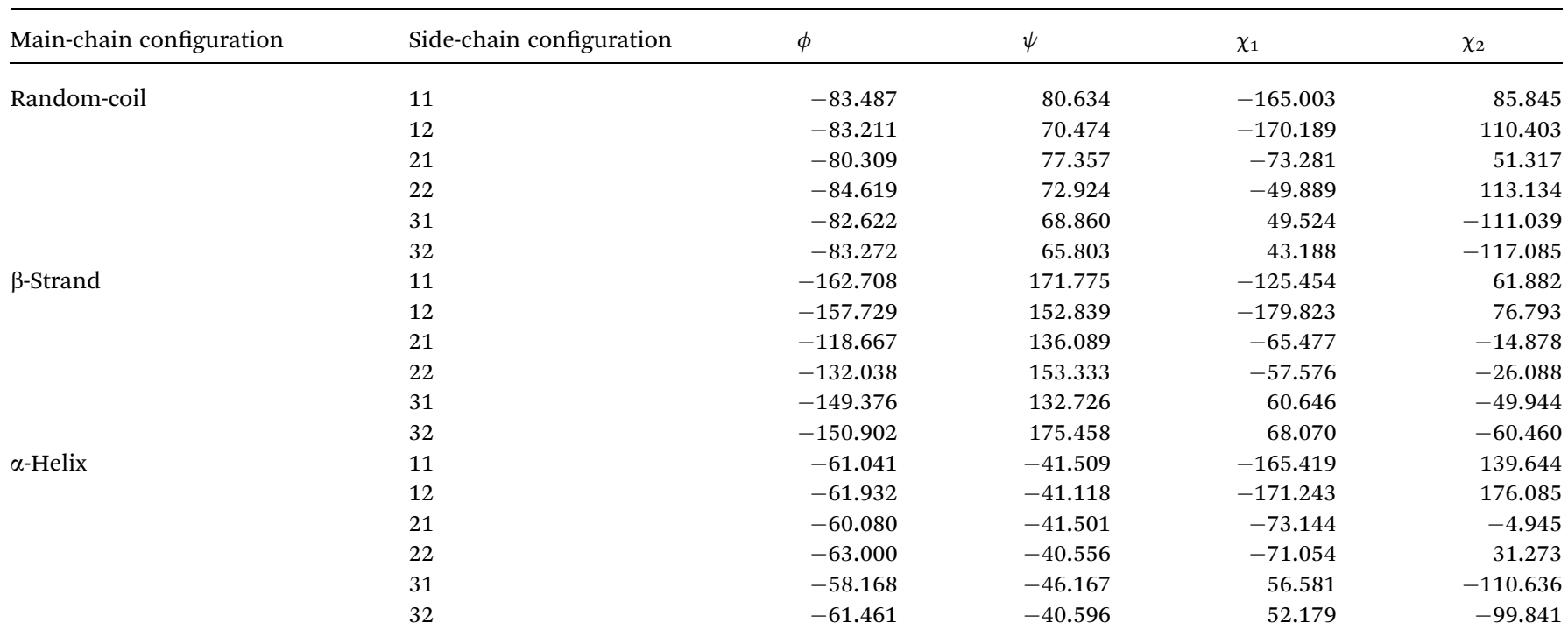

Table 2 Optimized Ramachandran dihedral angles $(\psi$ and $\phi)$ for the main-chain amide group in L-tyrosine and the corresponding angles $\left(\chi_{1}\right.$ and $\chi_{2}$ ) for the rotational conformers defining the residues (all in degree). Angles are reported for Tyr residues in random coil, $\alpha$-helix, $\beta$-strand configuration

\begin{tabular}{|c|c|c|c|c|c|}
\hline Main-chain configuration & Side-chain configuration & $\phi$ & $\psi$ & $\chi_{1}$ & $\chi_{2}$ \\
\hline \multirow[t]{6}{*}{ Random-coil } & 11 & -83.172 & 79.274 & -163.680 & 91.593 \\
\hline & 12 & -83.164 & 78.822 & -163.729 & 90.941 \\
\hline & 21 & -84.599 & 73.952 & -54.497 & -67.299 \\
\hline & 22 & -84.605 & 73.564 & -55.577 & 109.712 \\
\hline & 31 & -82.998 & 60.807 & 42.379 & -101.330 \\
\hline & 32 & -82.938 & 60.709 & 41.995 & -102.427 \\
\hline \multirow[t]{6}{*}{$\beta$-Strand } & 11 & -158.147 & 164.174 & -161.169 & 71.103 \\
\hline & 12 & -157.355 & 161.546 & -164.719 & 70.301 \\
\hline & 21 & -117.278 & 140.859 & -61.060 & 96.373 \\
\hline & 22 & -124.004 & 143.438 & -57.805 & 98.939 \\
\hline & 31 & -153.901 & 166.312 & 59.342 & -91.861 \\
\hline & 32 & -154.404 & 167.351 & 59.864 & -91.742 \\
\hline \multirow[t]{6}{*}{$\alpha$-Helix } & 11 & -56.374 & -41.444 & -179.701 & 109.164 \\
\hline & 12 & -55.709 & -41.421 & -179.407 & 89.914 \\
\hline & 21 & -58.532 & -40.313 & -48.846 & 97.869 \\
\hline & 22 & -58.947 & -40.619 & -43.136 & 102.711 \\
\hline & 31 & -55.253 & -41.267 & 54.833 & -75.374 \\
\hline & 32 & -53.901 & -41.385 & 61.283 & -89.946 \\
\hline
\end{tabular}

present, in a comparative fashion, the corresponding linear and nonlinear absorption and CD spectra of His11, Phe1, and Tyr11 residues calculated with CAM-B3LYP (the comparative plots of all the remaining His $\chi_{1} \chi_{2}$, Phe $\chi_{1}$, and Tyr $\chi_{1} \chi_{2}$ residues, calculated with CAM-B3LYP and B3LYP can be found in ESI $\dagger$ ). In order to facilitate the comparison between the linear and nonlinear spectra, OPA wavelength is used for both cases throughout the discussion of Fig. 9-11.

Before beginning the discussion in this section, it is worth noting that all three amino acids present more distinguishable and measurable variations in the nonlinear absorption spectra,
TPA and TPCD, of the different conformations than in their corresponding linear counterpart, OPA and ECD.

First, in the TPA spectra of His11 (Fig. 9) one can precisely identify its presence in all three different conformations through: (i) the longer wavelength band at $\sim 200 \mathrm{~nm}$ in the $\alpha$-helix, (ii) a double peak broad band between $140 \mathrm{~nm}$ and $160 \mathrm{~nm}$ in $\beta$-strand, and (iii) the very specific band in the blue side of the spectrum at $\sim 130 \mathrm{~nm}$ in random coil. On the other hand, in the TPCD spectra of the same amino acid residue, in all three different configurations, one can observe the following spectral fingerprints: (i) a strong negative band in the red side of 
Table 3 Optimized Ramachandran dihedral angles $(\psi$ and $\phi)$ for the main-chain amide group in L-phenylalanine and the corresponding angles $\left(\chi_{1}\right.$ and $\left.\chi_{2}\right)$ for the rotational conformers defining the residues (all in degree). Angles are reported for Phe residues in random coil, $\alpha$-helix, $\beta$-strand configuration

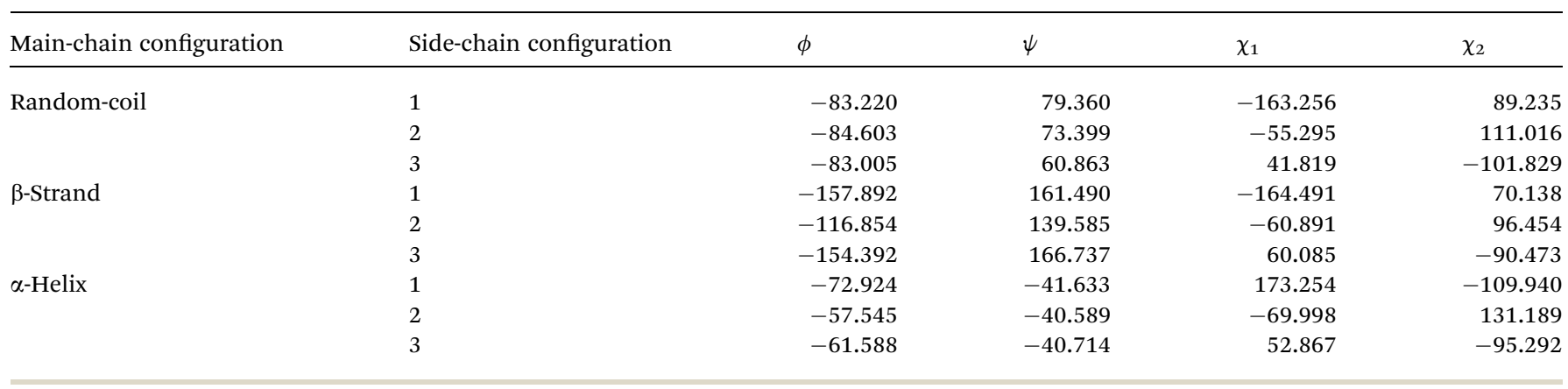
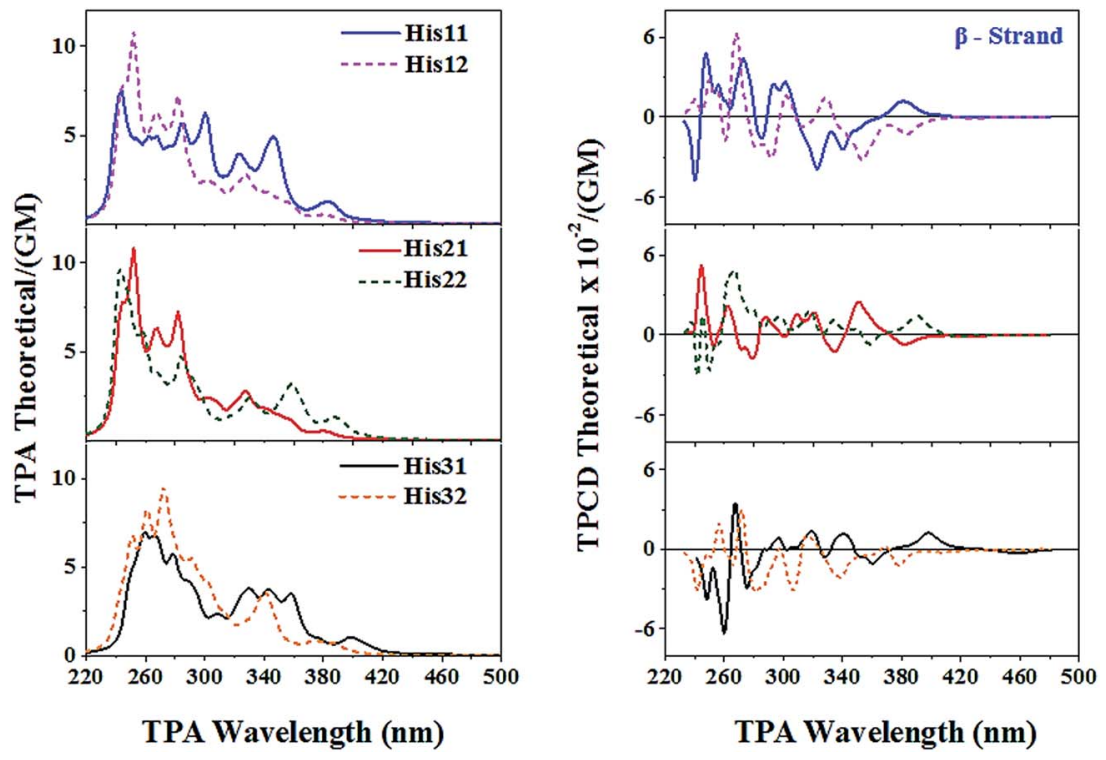

Fig. 2 Comparative plots of TPA (left) and TPCD (right) spectra of L-histidine models in $\beta$-strand configuration. His11 and His12 (top), His21 and His22 (middle), His31 and His32 (bottom). TPA and TPCD response for the lowest 80 electronic excited states of all optimized structures were computed with TD-DFT/CAM-B3LYP/6-311G(d) in gas phase using Dalton 2011.32

the spectrum at $\sim 200 \mathrm{~nm}$ in $\alpha$-helix, (ii) a specific negative double band between $160 \mathrm{~nm}$ and $180 \mathrm{~nm}$ in $\beta$-strand, and (iii) a clearly strong double peak band between $150 \mathrm{~nm}$ and $170 \mathrm{~nm}$ in random coil. The presence of these specific bands, that allows the recognition of the different configurations using His residues, reveals the applicability of TPCD as a complementary technique to ECD for the study of complex protein structures. However, the most important point to be highlighted on this part of the analysis is the number of unique and identifiable spectral signatures of His11 in all three configurations down to $110 \mathrm{~nm}$. These features provide additional conformational information to the limited traditional ECD technique. In fact, inspecting the OPA and ECD spectra of His11 in $\alpha$-helix, $\beta$-strand, and random coil, in the short wavelengths range $(<180 \mathrm{~nm})$ one can see a very large number of convoluted bands overlapping with each other through almost the whole spectrum. Only in the ECD plot, and within the typical spectral range employed for the study of proteins using linear spectroscopy, i.e. above $180 \mathrm{~nm}$, one can observe few spectral signatures for $\beta$-strand and random coil useful for the identification of this amino acid in protein secondary structures (shaded area).

Second, in Phe1 (Fig. 10) one can distinguish similar characteristics to those observed for His11, i.e. less intricate nonlinear spectra down to $110 \mathrm{~nm}$ and the presence of specific bands for each conformation, mostly in the TPCD plot, that allows identification of one from another. Although, in TPA one can only distinguish the $\beta$-strand from the other two configurations through a strong peak at $\sim 165 \mathrm{~nm}$, in TPCD characteristic signals with opposite sign allow identification of all three conformations independently. For instance, while $\beta$ strand presents a unique negative broad TPCD band between $150 \mathrm{~nm}$ and $180 \mathrm{~nm}$, the other two configurations present a positive one. To differentiate between $\alpha$-helix and random coil 

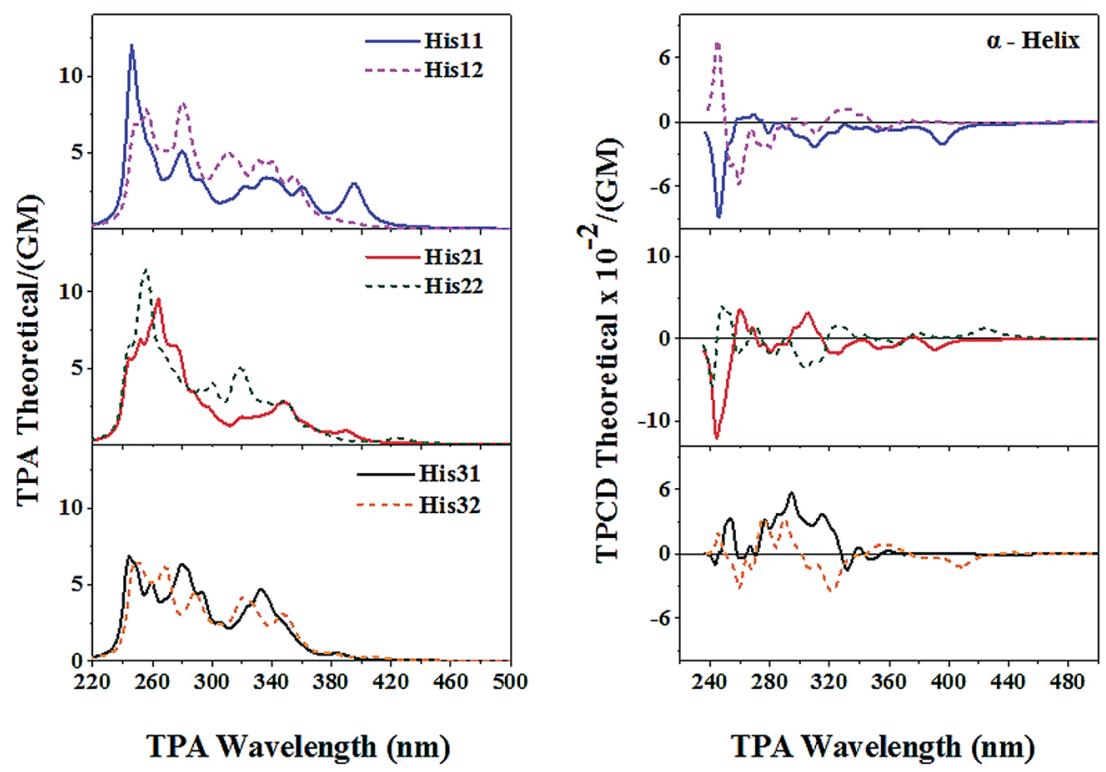

Fig. 3 Comparative plots of TPA (left) and TPCD (right) spectra of L-histidine models in $\alpha$-helix configuration. His11 and His12 (top), His21 and His22 (middle), His31 and His32 (bottom). TPA and TPCD response for the lowest 80 electronic excited states of all optimized structures were computed with TD-DFT/CAM-B3LYP/6-311G(d) in gas phase using Dalton 2011.32
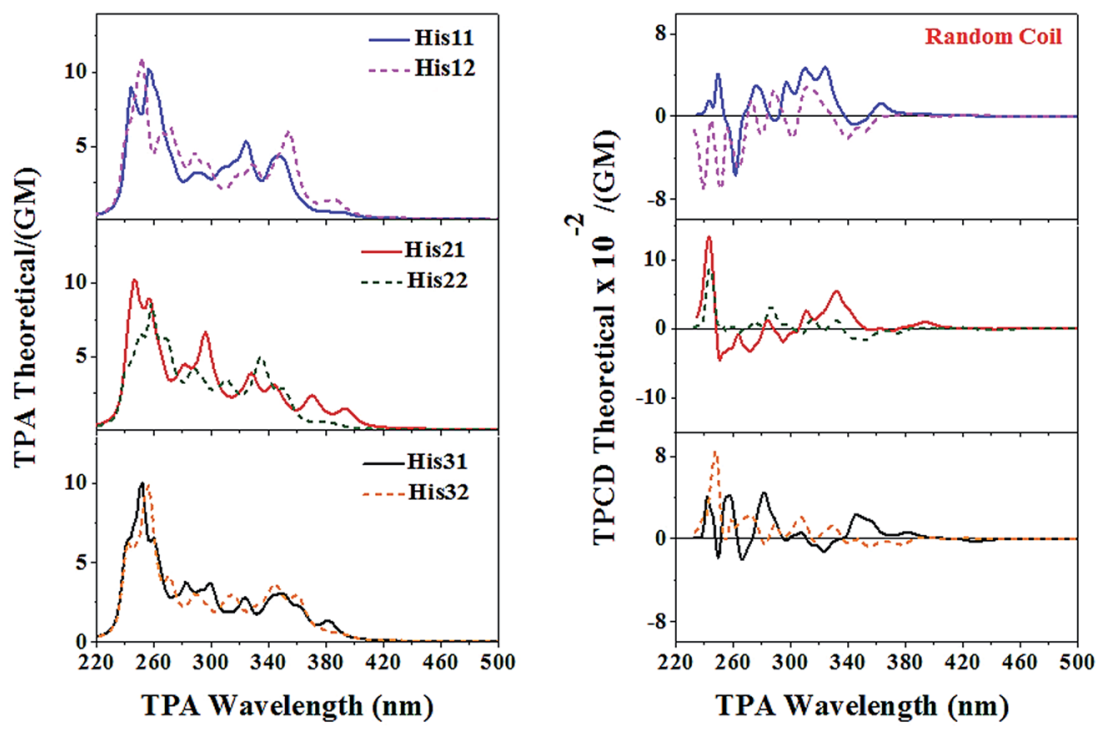

Fig. 4 Comparative plots of TPA (left) and TPCD (right) spectra of L-histidine models in random coil configuration. His11 and His12 (top), His21 and His22 (middle), His31 and His32 (bottom). TPA and TPCD response for the lowest 80 electronic excited states of all optimized structures were computed with TD-DFT/CAM-B3LYP/6-311G(d) in gas phase using Dalton 2011.32

one should look at longer wavelengths (small positive band at $\sim 175 \mathrm{~nm}$ ) for the former and at very short wavelengths (sharp negative peak at $\sim 125 \mathrm{~nm}$ ) for the latter. Again, the most remarkable aspects of this analysis are, (i) the unique and identifiable spectral signatures in all three configurations down to $110 \mathrm{~nm}$, and (ii) the accessibility to additional conformational information in the FUV. Even in the event were ECD becomes applicable for the conformational study of proteins in the FUV, the number of convoluted bands, overlapping with each other in that region, would limit its use in that spectral region - ECD is truly functional above $\sim 180 \mathrm{~nm}$.

Third, besides the already discussed characteristic nonlinear absorption fingerprints observed in His and Phe, we found that TPA in Tyr (Fig. 11) is not reliable for the differentiation between main-chain "back-bone" conformations. However, TPCD shows obvious differences in certain spectral regions to specifically identify all three conformations: (i) $\alpha$-helix: negative broad band in the red side of the spectrum above $170 \mathrm{~nm}$, (ii) $\beta$-strand: positive sharp peak at $\sim 125 \mathrm{~nm}$, and (iii) random coil: positive 

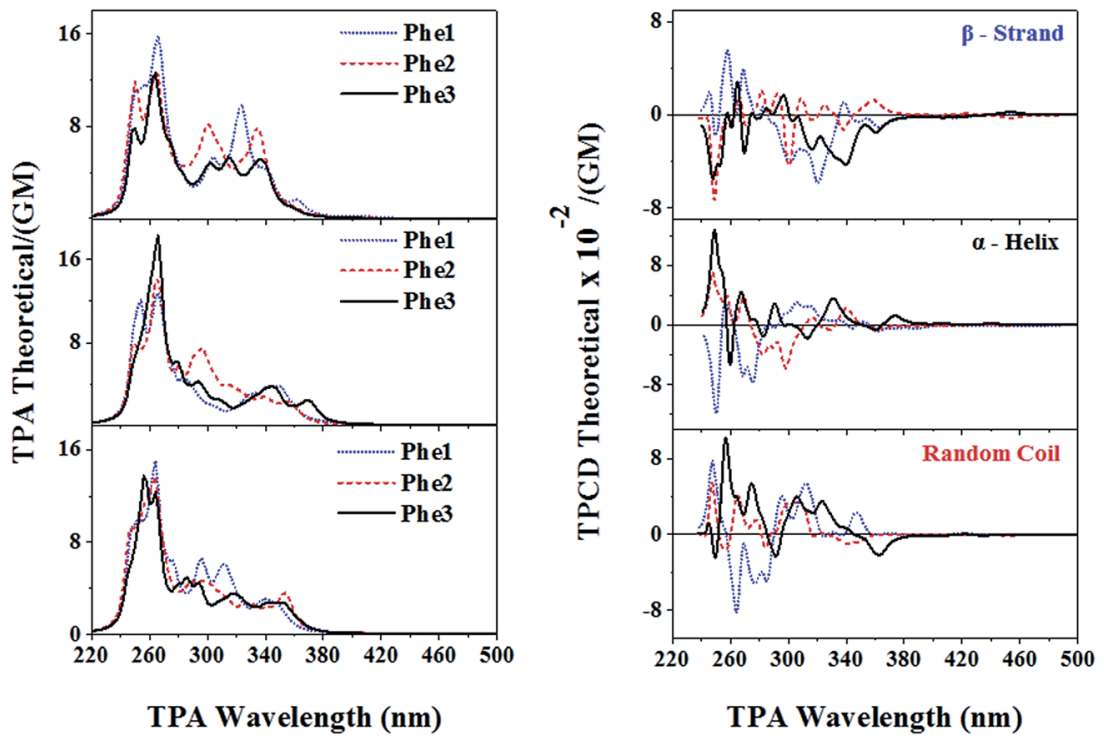

Fig. 5 Comparative plots of TPA (left) and TPCD (right) spectra of L-phenylalanine models in $\beta$-strand (top), $\alpha$-helix (middle), and random coil (bottom) configuration. TPA and TPCD response for the lowest 80 electronic excited states of all optimized structures were computed with TD-DFT/CAM-B3LYP/6-311G(d) in gas phase using Dalton 2011.32
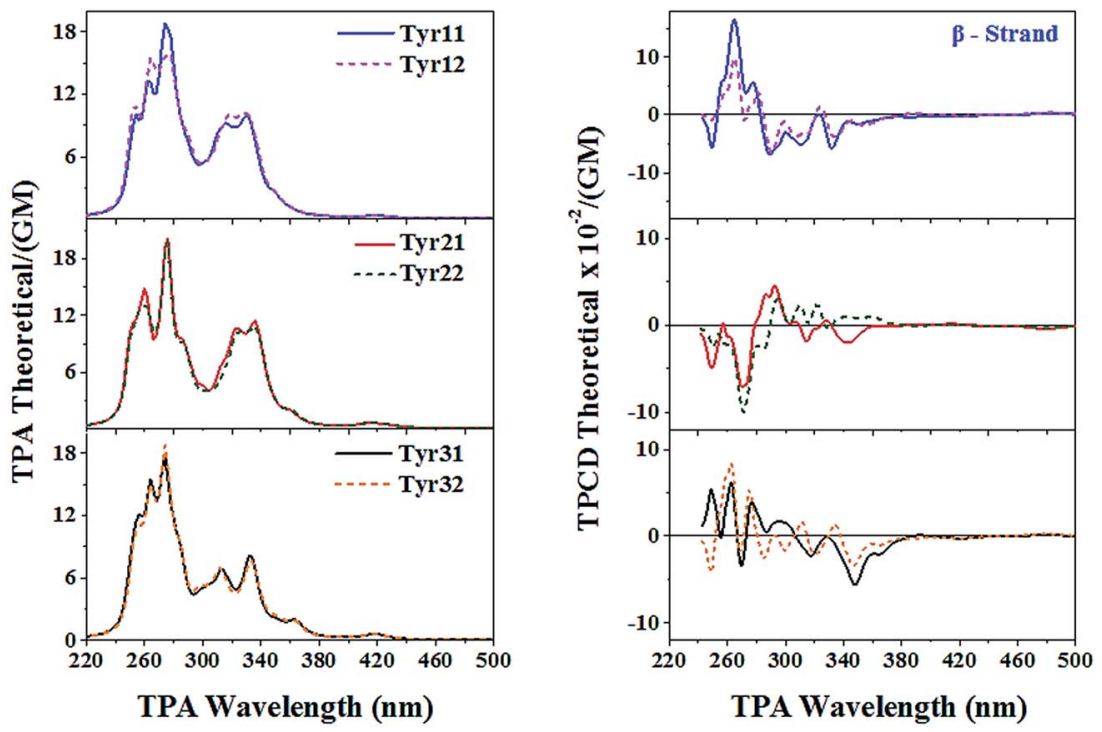

Fig. 6 Comparative plots of TPA (left) and TPCD (right) spectra of L-tyrosine models in $\beta$-strand configuration. Tyr11 and Tyr12 (top), Tyr21 and Tyr22 (middle), Tyr31 and Tyr32 (bottom). TPA and TPCD response for the lowest 80 electronic excited states of all optimized structures were computed with TD-DFT/CAM-B3LYP/6-311G(d) in gas phase using Dalton $2011 .{ }^{32}$

broad band between $140 \mathrm{~nm}$ and $170 \mathrm{~nm}$. One more time, it has been proven that TPCD has all the potential as a tool for the identification between $\alpha$-helix, $\beta$-strand, and random coil utilizing aromatic amino acid residues.

Finally, we present the direct comparison of the TPA and TPCD spectra on a set of two amino acid residues of His, Phe and Tyr (His11-Phe1-Tyr11 and His21-Phe2-Tyr21), in their corresponding $\beta$-strand, $\alpha$-helix, and random coil configurations. As it can be observed in Fig. 12-14, they all exhibit clear spectral differences in TPA and TPCD, in each configuration.
These signatures could be used, synergistically, for the conformational analysis of peptides and proteins in the FUV.

First, we assess the potential of TPA spectroscopy for the conformational analysis of peptides and proteins examining the specific spectral signatures of aromatic amino acids in different configurations. In $\beta$-strand, one can observe, simultaneously, the following complementary spectral fingerprints: a strong peak at $\sim 270 \mathrm{~nm}$ in Tyr11, a small band at $\sim 380 \mathrm{~nm}$ in His11, a sharp peak at $\sim 280 \mathrm{~nm}$ in Tyr21, and a relatively intense band at $\sim 290$ in Phe $2 \mathrm{~nm}$. In $\alpha$-helix, one can notice the following 

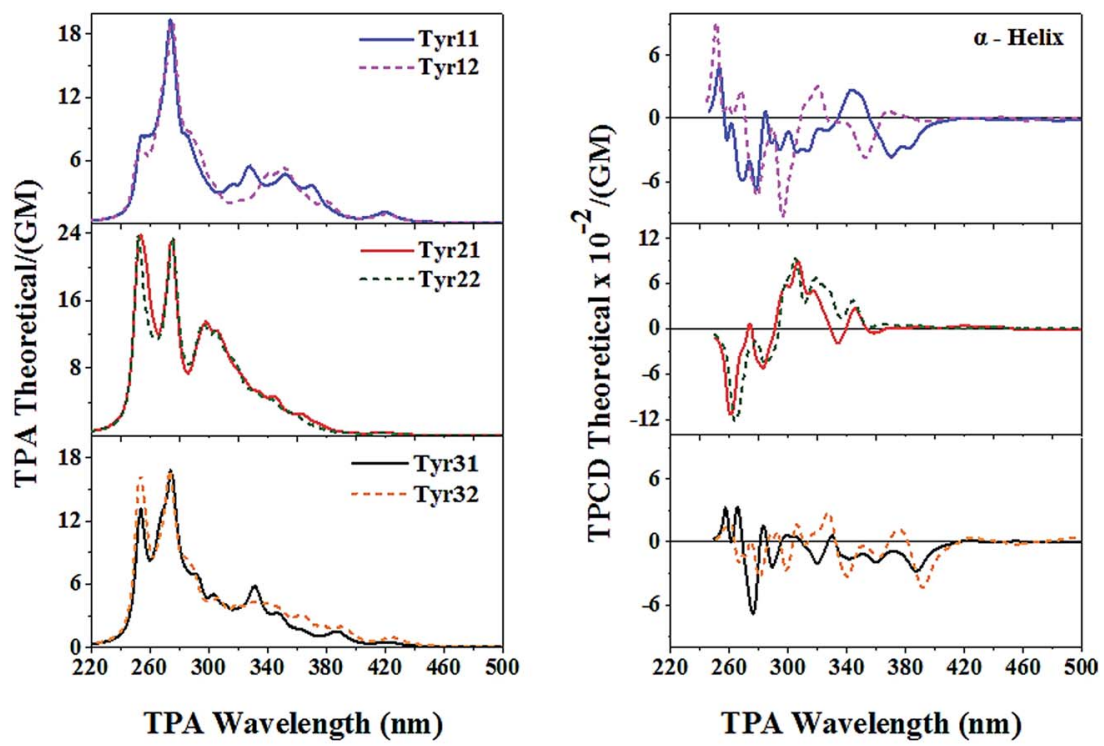

Fig. 7 Comparative plots of TPA (left) and TPCD (right) spectra of L-tyrosine models in $\alpha$-helix configuration. TPA and TPCD response for the lowest 80 electronic excited states of all optimized structures were computed with TD-DFT/CAM-B3LYP/6-311G(d) in gas phase using Dalton $2011 .^{32}$
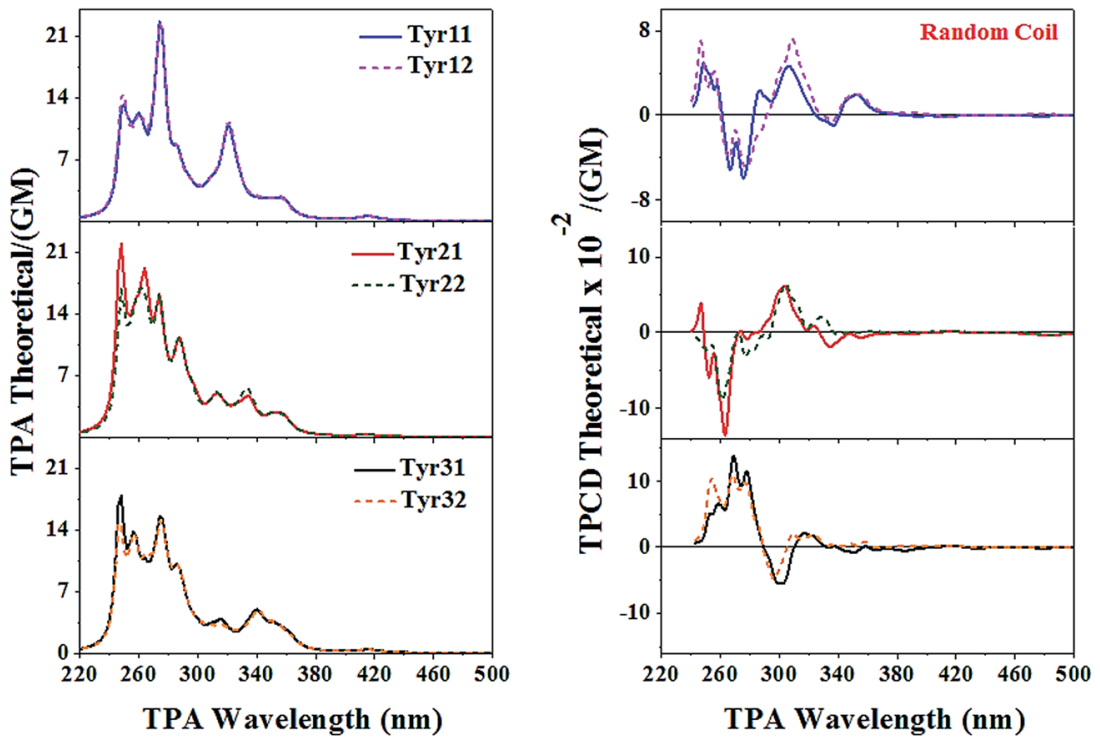

Fig. 8 Comparative plots of TPA (left) and TPCD (right) spectra of L-tyrosine models in random coil configuration. Tyr11 and Tyr12 (top), Tyr21 and Tyr22 (middle), Tyr31 and Tyr32 (bottom). TPA and TPCD response for the lowest 80 electronic excited states of all optimized structures were computed with TD-DFT/CAM-B3LYP/6-311G(d) in gas phase using Dalton 2011.32

complementary spectral features: a strong peak at $\sim 270 \mathrm{~nm}$ in Tyr11, a small band at $\sim 390 \mathrm{~nm}$ in His11, and a broad band with two strong peaks at $\sim 260 \mathrm{~nm}$ and $280 \mathrm{~nm}$ in Tyr21. In random coil one can distinguish the following spectral signatures: two strong peaks centered at $\sim 280 \mathrm{~nm}$ and $330 \mathrm{~nm}$ in Tyr11, a very broad band with defined spectral structure between $240 \mathrm{~nm}$ and $300 \mathrm{~nm}$ in Tyr21, and a weak double band above $\sim 360 \mathrm{~nm}$ in His21. Although, some peaks/bands are similar for different amino acid residues in different configurations, several others are very specific for each structural arrangement. This outcome shows the limited but still useful potential of TPA for the targeted analysis.

Next, we present the examination of the TPCD spectra of the same amino acid residues, in the same protein secondary structures. As shown subsequently, our results reveal the exceptional spectroscopic ability of TPCD, compared to the traditional ECD, for the analysis of protein structures in a region where the overcrowded ECD spectra make the identification of complex structures using linear spectroscopy very difficult. ${ }^{24}$ 

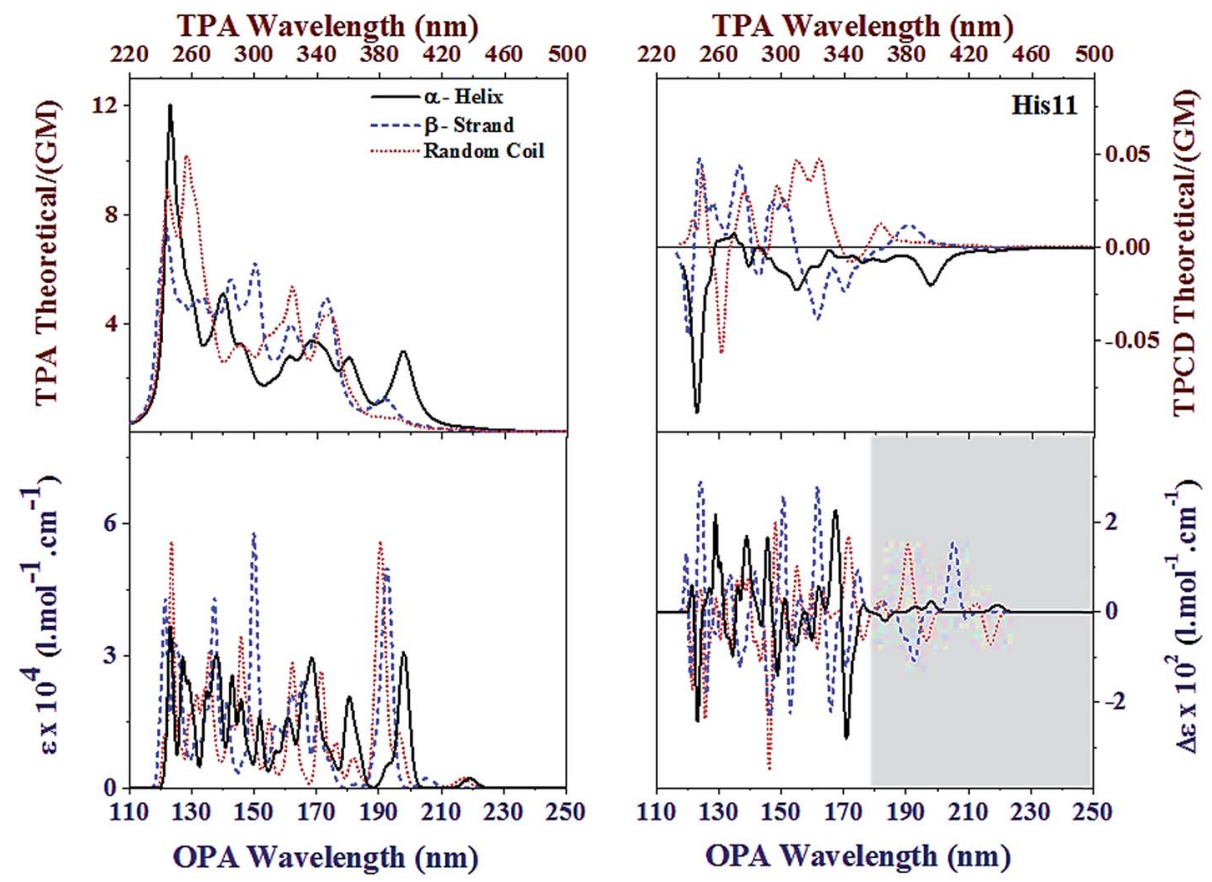

Fig. 9 Comparative plots of TPA (top left), TPCD (top right), OPA (bottom left), and ECD (bottom right) spectra of His11 in random coil (red dotted line), $\alpha$-helix (black solid line) and $\beta$-strand (blue dashed line) configuration. TPA and TPCD response for the lowest 80 electronic excited states of all optimized structures were computed with TD-DFT/CAM-B3LYP/6-311G(d) in gas phase using Dalton 2011. ${ }^{32}$ OPA and ECD for the lowest 80 electronic excited states were computed of all optimized structures at the CAM-B3LYP/6-311G(d) level of theory using Gaussian 09 (ref. 31) in gas phase. Shaded area indicates where ECD is truly functional.
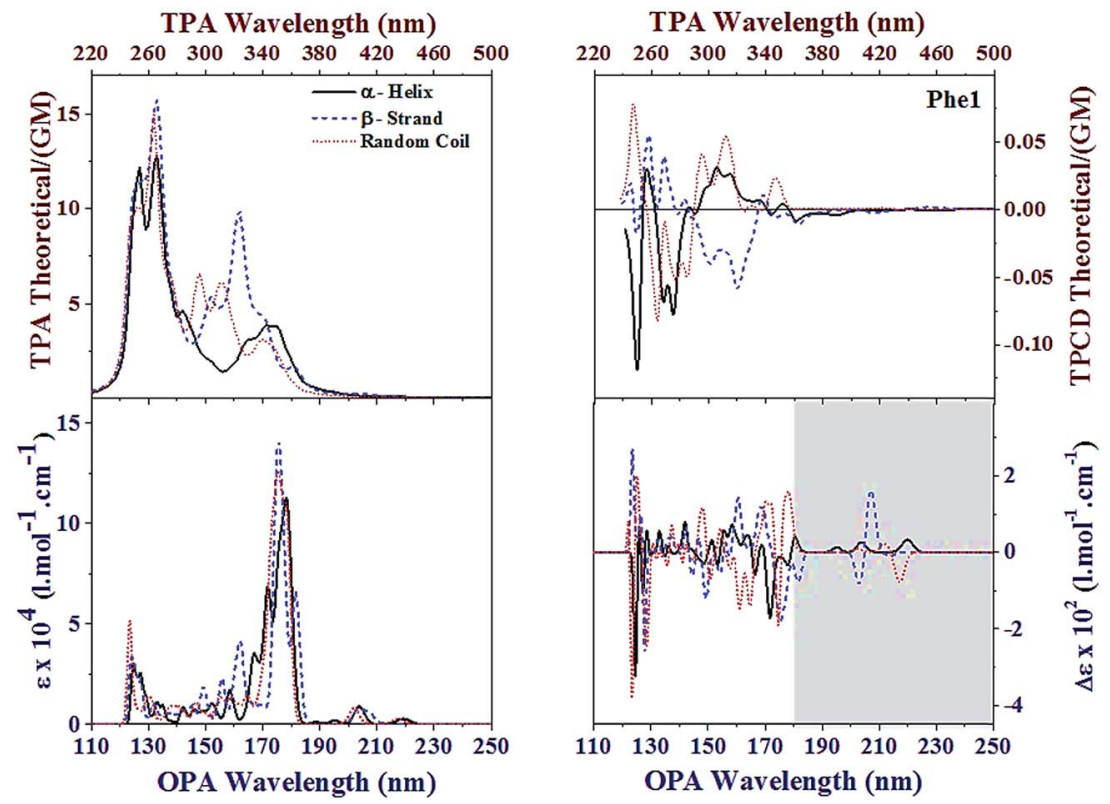

Fig. 10 Comparative plots of TPA (top left), TPCD (top right), OPA (bottom left), and ECD (bottom right) spectra of Phe1 in random coil (red dotted line), $\alpha$-helix (black solid line) and $\beta$-strand (blue dashed line) configuration. TPA and TPCD response for the lowest 80 electronic excited states of all optimized structures were computed with TD-DFT/CAM-B3LYP/6-311G(d) in gas phase using Dalton 2011. ${ }^{32}$ OPA and ECD for the lowest 80 electronic excited states were computed of all optimized structures at the CAM-B3LYP/6-311G(d) level of theory using Gaussian 09 (ref. 31) in gas phase. Shaded area indicates where ECD is truly functional. 

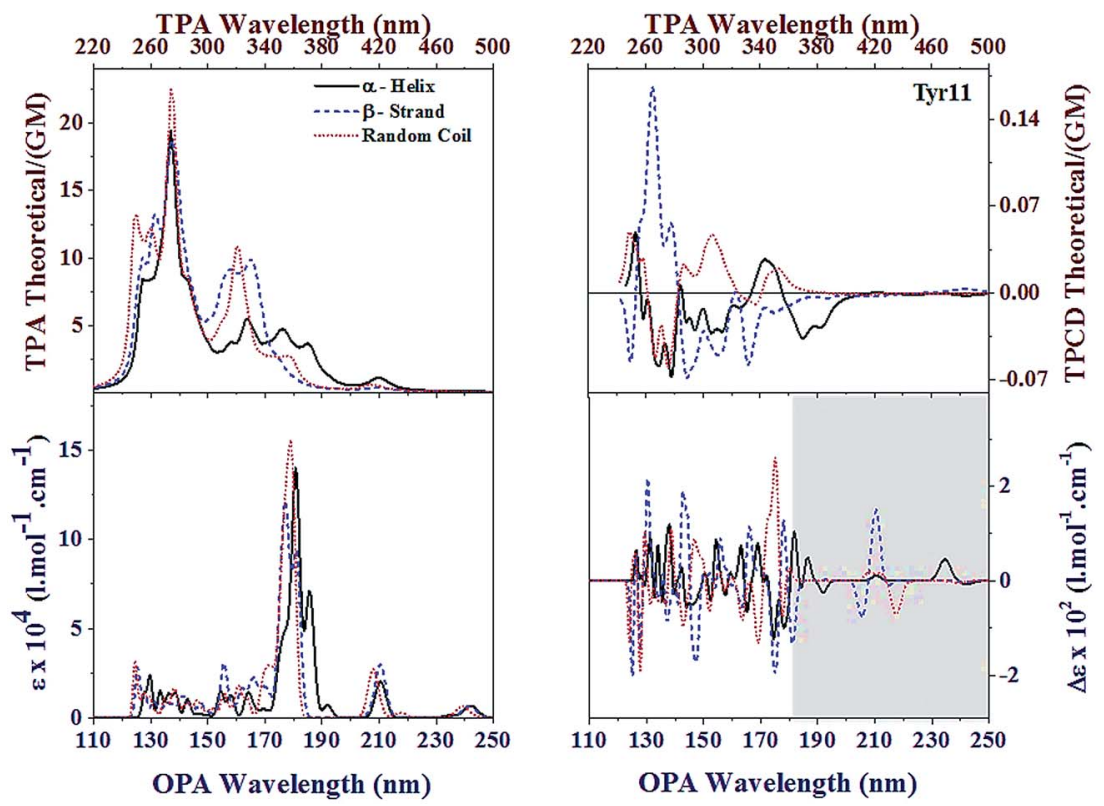

Fig. 11 Comparative plots of TPA (top left), TPCD (top right), OPA (bottom left), and ECD (bottom right) spectra of Tyr11 in random coil (red dotted line), $\alpha$-helix (black solid line) and $\beta$-strand (blue dashed line) configuration. TPA and TPCD response for the lowest 80 electronic excited states of all optimized structures were computed with TD-DFT/CAM-B3LYP/6-311G(d) in gas phase using Dalton 2011.32 OPA and ECD for the lowest 80 electronic excited states were computed of all optimized structures at the CAM-B3LYP/6-311G(d) level of theory using Gaussian 09 (ref. 31) in gas phase. Shaded area indicates where ECD is truly functional.
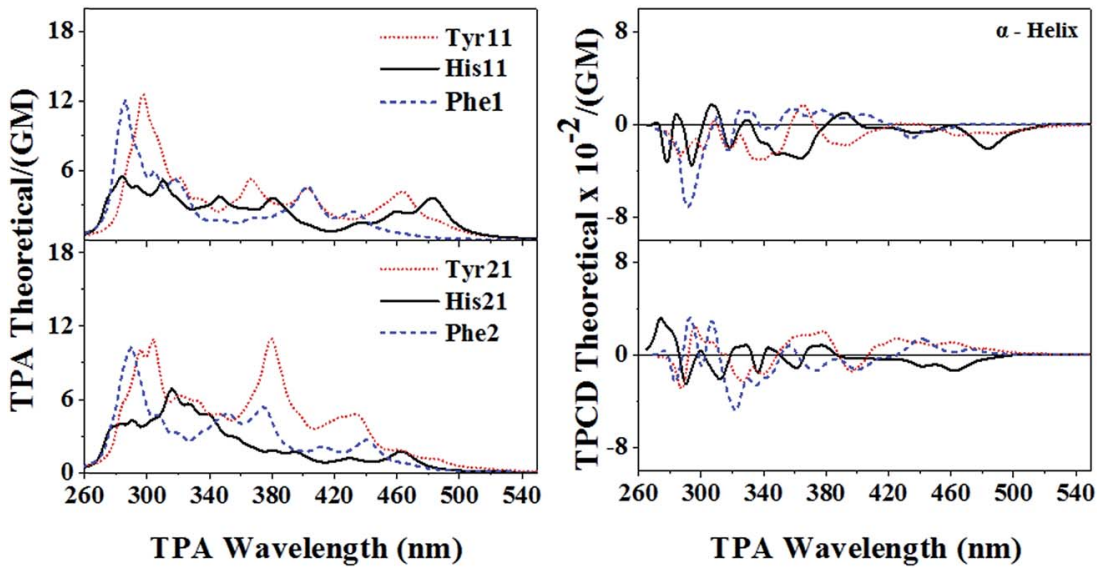

Fig. 12 Comparative plots of TPA (left) and TPCD (right) spectra of Tyr11 (red dotted line), His11 (black solid line) and Phe1 (blue dashed line) (top), and Tyr21 (red dotted line), His21 (black solid line) and Phe2 (blue dashed line) (bottom), in $\beta$-strand configuration. TPA and TPCD response for the lowest 80 electronic excited states of all optimized structures were computed with TD-DFT/CAM-B3LYP/6-311G(d) in gas phase using Dalton $2011 .{ }^{32}$

Using TPA one can attempt to differentiate between protein secondary structures by looking for the following specific fingerprints in Tyr, i.e. (a) $\beta$-strand: strong band centered at $\sim 280 \mathrm{~nm}$ with two shoulders on the blue side of the spectrum in Tyr11; (b) $\alpha$-helix: broad band with two sharp peaks at $\sim 250 \mathrm{~nm}$ and $280 \mathrm{~nm}$ in Tyr21; (c) random coil: strong broad band with structure between $\sim 240 \mathrm{~nm}$ and $300 \mathrm{~nm}$ in Tyr21. Other amino acid residues do not present such clear and differentiable signatures in TPA.

Using TPCD one can clearly gather conformational information from all three amino acids. For instance, $\beta$-strand can selectively be identified through specific positive or negative signatures at $\sim 260 \mathrm{~nm}$ in Tyr11, $270 \mathrm{~nm}$ in Tyr21, $300 \mathrm{~nm}$ in His11 and $290 \mathrm{~nm}$ in Phe1; (b) $\alpha$-helix through spectral bands at $\sim 360 \mathrm{~nm}$ in Tyr11, $240 \mathrm{~nm}$ in His21 and in Phe2; (c) Random coil by measuring TPCD at $\sim 260 \mathrm{~nm}$ and $300 \mathrm{~nm}$ in Tyr11, 280 $\mathrm{nm}$ and $330 \mathrm{~nm}$ in His11. TPCD exposes its remarkable capability for the identification of molecules within the same protein structure using specific signatures of different aromatic amino acids, simultaneously. 

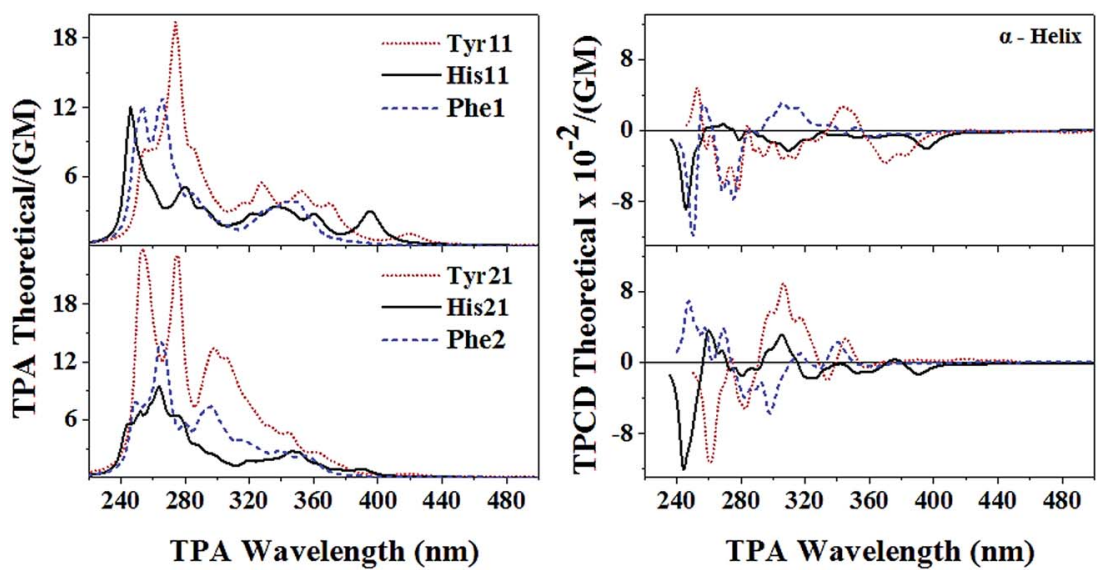

Fig. 13 Comparative plots of TPA (left) and TPCD (right) spectra of Tyr11 (red dotted line), His11 (black solid line) and Phe1 (blue dashed line) (top), and Tyr21 (red dotted line), His21 (black solid line) and Phe2 (blue dashed line) (bottom), in $\alpha$-helix configuration. TPA and TPCD response for the lowest 80 electronic excited states of all optimized structures were computed with TD-DFT/CAM-B3LYP/6-311G(d) in gas phase using Dalton $2011 .^{32}$
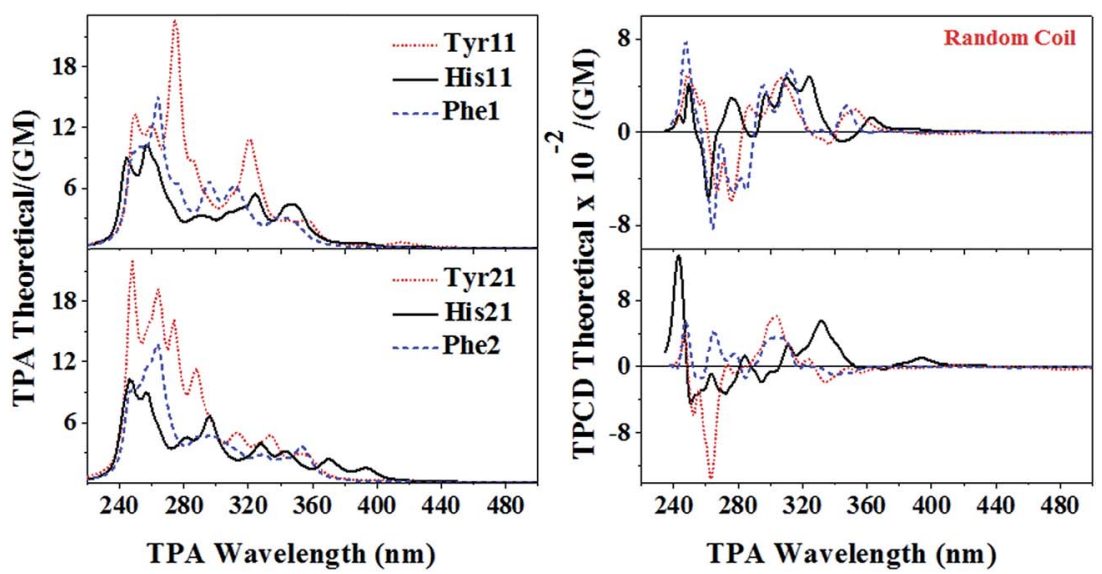

Fig. 14 Comparative plots of TPA (left) and TPCD (right) spectra of Tyr11 (red dotted line), His11 (black solid line) and Phe1 (blue dashed line) (top), and Tyr21 (red dotted line), His21 (black solid line) and Phe2 (blue dashed line) (bottom), in random coil configuration. TPA and TPCD response for the lowest 80 electronic excited states of all optimized structures were computed with TD-DFT/CAM-B3LYP/6-311G(d) in gas phase using Dalton $2011 .^{32}$

\section{Conclusions}

The potential of TPCD for the study and analysis of complex chemical structures such as peptides and proteins in a region that is impenetrable using linear absorption processes was demonstrated. The specific signatures found in L-histidine, L-phenylalanine, and L-tyrosine residues, in $\beta$-strand, $\alpha$-helix, and random coil configurations, validated the distinguishing ability of TPCD to differentiate between: (i) very similar species with equal $\chi_{1}$, and (ii) between equal or dissimilar species in different conformational configuration. The evaluation of the TPCD spectra of the re-optimized structures demonstrated that the main-chain angles are undeniably influenced by the sidechain configuration and vice versa. Through the determination of the TPCD signal of multiple amino acids simultaneously one can gather multi-parametric data to certify the existence of a specific conformation and assess a small distortion of the same in the FUV. Finally, we should mention that in some cases the TPA spectra provide additional conformational information that can be used for the study of amino acid residues.

\section{Acknowledgements}

This work was partially supported by the National Science Foundation through Grant Number CHE-0840431. The computing time provided by STOKES ARCC is gratefully acknowledged. The authors would like to thank N.I.K.O. for his assistance for the completion of this work on time.

\section{References}

1 R. Schweitzer-Stenner, J. Phys. Chem. B, 2004, 108, 1696516975. 
2 S. M. Kelly, T. J. Jess and N. C. Price, Biochim. Biophys. Acta, Proteins Proteomics, 2005, 1751, 119-139.

3 N. J. Greenfield, TrAC, Trends Anal. Chem., 1999, 18, 236-244. 4 S. Y. Venyaminov and J. T. Yang, in Circular Dichroism and the Conformational Analysis of Biomolecules, ed. G. D. Fasman, Plenum Press, LLC, New York, 1996, ch. 3, pp. 69-108.

5 N. Berova, P. L. Polavarapu, K. Nakanishi and R. W. Woody, Comprehensive Chiroptical Spectroscopy, John Wiley \& Sons, Hoboken, NJ, 2012.

6 R. W. Woody and K. A. Dunker, in Circular Dichroism and the Conformational Analysis of Biomolecules, ed. G. D. Fasman, Plenum Press, New York, 1996, pp. 109-158.

7 E. H. Strickland, Crit. Rev. Biochem., 1974, 2, 113-175.

8 P. C. Kahn, Methods Enzymol., 1979, 61, 339-378.

9 A. J. Miles and B. A. Wallace, Chem. Soc. Rev., 2006, 35, 39-51.

10 T. Tanaka, T. S. Kodama, H. E. Morita and T. Ohno, Chirality, 2006, 18, 652-661.

11 E. Runge and E. K. U. Gross, Phys. Rev. Lett., 1984, 52, 9971000.

12 G. N. Ramachandran, C. Ramakrishnan and V. Sasisekharan, J. Mol. Biol., 1963, 7, 95-99.

13 C. Toro, L. De Boni, N. Lin, F. Santoro, A. Rizzo and F. E. Hernandez, Chem.-Eur. J., 2010, 16, 3504-3509.

14 I. Tinoco, J. Chem. Phys., 1975, 62, 1006-1009.

15 E. A. Power, J. Chem. Phys., 1975, 63, 1348-1350.

16 D. L. Andrews, Chem. Phys., 1976, 16, 419-424.

17 B. Jansik, A. Rizzo and H. Agren, Chem. Phys. Lett., 2005, 414, 461-467.

18 L. De Boni, C. Toro and F. E. Hernández, Opt. Lett., 2008, 33, 2958-2960.

19 R. W. Boyd, Nonlinear Optics, Academic Press, San Diego, CA, 1992.

20 W. Denk, J. Strickler and W. Webb, Science, 1990, 248, 73-76.

21 J. M. Squirrell, D. L. Wokosin, J. G. White and B. D. Bavister, Nat. Biotechnol., 1999, 17, 763-767.

22 J. R. Starkey, A. K. Rebane, M. A. Drobizhev, F. Meng, A. Gong, A. Elliott, K. McInnerney and C. W. Spangler, Clin. Cancer Res., 2008, 14, 6564-6573.

23 B. Jansik, A. Rizzo and H. Agren, J. Phys. Chem. B, 2007, 111, 446-460.

24 Y. Vesga, C. Diaz, M. Higgs and F. E. Hernandez, Chem. Phys. Lett., 2014, 601, 6-12.
25 M. Dierksen and S. Grimme, J. Chem. Phys., 2006, 124, 174301.

26 A. D. Becke, Phys. Rev. A, 1988, 38, 3098-3100.

27 A. D. Becke, J. Chem. Phys., 1993, 98, 5648-5652.

28 C. Lee, W. Yang and R. G. Parr, Phys. Rev. B: Condens. Matter, 1988, 37, 785-789.

29 R. Krishnan, J. S. Binkley, R. Seeger and J. A. Pople, J. Chem. Phys., 1980, 72, 650-654.

30 A. D. McLean and G. S. Chandler, J. Chem. Phys., 1980, 72, 5639-5648.

31 M. J. Frisch, G. W. Trucks, H. B. Schlegel, G. E. Scuseria, M. A. Robb, J. R. Cheeseman, G. Scalmani, V. Barone, B. Mennucci and G. A. Petersson, Gaussian, Inc., Wallingford CT, 2009.

32 K. Aidas, C. Angeli, K. L. Bak, V. Bakken, R. Bast, L. Boman, O. Christiansen, R. Cimiraglia, S. Coriani, P. Dahle and E. K. Dalskov, Wiley Interdiscip. Rev.: Comput. Mol. Sci., 2014, 4(3), 269-284.

33 W. M. McClain, J. Chem. Phys., 1971, 55, 2789-2796.

34 M. G. Vivas, C. Diaz, L. Echevarria, C. R. Mendonca, F. E. Hernández and L. De Boni, J. Phys. Chem. B, 2013, 117, 2742-2747.

35 D. L. Silva, N. A. Murugan, J. Kongsted, Z. Rinkevicius, S. Canuto and H. Agren, J. Phys. Chem. B, 2012, 116, 81698181.

36 M. Guillaume, K. Ruud, A. Rizzo, S. Monti, Z. Lin and X. Xu, J. Phys. Chem. B, 2010, 114, 6500-6512.

37 U. J. Meierhenrich, J.-J. Filippi, C. Meinert, J. H. Bredehöft, J.-i. Takahashi, L. Nahon, N. C. Jones and S. V. Hoffmann, Angew. Chem., Int. Ed., 2010, 49, 7799-7802.

38 N. Lin, F. Santoro, X. Zhao, C. Toro, L. De Boni, F. E. Hernández and A. Rizzo, J. Phys. Chem. B, 2011, 115, 811-824.

39 K. Kristensen, J. Kauczor, T. Kjærgaard and P. Jørgensen, J. Chem. Phys., 2009, 131, 044112.

40 V. Barone, Computational Strategies for Spectroscopy: From Small Molecules to Nano Systems, John Wiley \& Sons, Hoboken, NJ, 2012.

41 K. Kristensen, J. Kauczor, A. J. Thorvaldsen, P. Jørgensen, T. Kjærgaard and A. Rizzo, J. Chem. Phys., 2011, 134, 214104. 